\title{
Radiofrequency ablation for treatment of refractory gastric antral vascular ectasia (with video)
}

Authors

Institutions
Tanima Jana' ${ }^{1}$, Nirav Thosani ${ }^{1,3}$, Michael B. Fallon ${ }^{1,2}$, Andrew W. Dupont ${ }^{1,2}$, Atilla Ertan ${ }^{1,2}$

Division of Gastroenterology, Hepatology and Nutrition, Department of Internal Medicine, The University of Texas Health Science Center at Houston, Houston, Texas, United States

2 Ertan Digestive Disease Center, Memorial Hermann Hospital, Houston, Texas, United States

Division of Gastroenterology \& Hepatology, Stanford University School of Medicine, Stanford, California, United States submitted

10. November 2014 accepted after revision 21. November 2014

\section{Bibliography}

DOI http://dx.doi.org/

10.1055/s-0034-1391323

Published online: 11.2.2015

Endoscopy International Open

2015; 03: E125-E127

(c) Georg Thieme Verlag KG

Stuttgart - New York

E-ISSN 2196-9736

\section{Corresponding author \\ Nirav Thosani}

Division of Gastroenterology \& Hepatology

Stanford University School of Medicine

300 Pasteur Drive, MC: 5244

Stanford, California 94305

United States

Fax: +1-640-498-5174

ncthosani@gmail.com
Background and study aims: Gastric antral vascular ectasia (GAVE) is a known cause of gastrointestinal bleeding and chronic iron deficiency anemia. Endoscopic therapy with argon plasma coagulation (APC) is widely used for treatment of GAVE, but most patients continue to require repeated blood transfusions and multiple endoscopic procedures (refractory GAVE). We describe our initial experience regarding safety and efficacy of radiofrequency ablation (RFA) therapy in treating patients with refractory GAVE.

Patients and methods: We prospectively enrolled seven patients with refractory GAVE who had multiple prior treatments with APC. These patients were treated with RFA (HALO90 ULTRA Ablation Catheter System; Covidien, GI Solutions, Sunnyvale, CA) at Ertan Digestive Disease Center at our tertiary University Hospital.

\section{Abbreviations \\ $\nabla$ \\ APC argon plasma coagulation \\ AVM arteriovenous malformation \\ GAVE gastric antral vascular ectasia \\ IQR interquartile range \\ RFA radiofrequency ablation}

\section{Introduction \\ $\nabla$}

Gastric antral vascular ectasia (GAVE) is characterized by the presence of fragile, dilated, ectatic vessels within the superficial submucosa and mucosa of the stomach, predominantly in the antrum [1]. GAVE was first described in 1953 by Rider et al. as erosive gastritis with venocapillary ectasia [2]. GAVE is a known cause of upper gastrointestinal bleeding and often manifests as an iron deficiency anemia requiring frequent blood transfusion [3-6]. The characteristic endoscopic findings of longitudinal antral folds with tortuous red, ectatic vessels are pathognomonic for the
Results: Seven patients underwent a total of 12 RFA procedures for treatment of refractory GAVE. The median number of RFA procedures was 2 (Range, 1-3). Average pre- and post-procedural hemoglobin were $9.3 \mathrm{~g} / \mathrm{dL}$ and $10.16 \mathrm{~g} / \mathrm{dL}$, respectively. Five of seven patients (71\%) were transfusion-free after the RFA treatments while two patients continued to require blood transfusions. There were no complications in this series.

Conclusion: RFA can be an effective alternative to APC for treatment of GAVE refractory to previous endoscopic therapy. Additional studies are needed to identify which subset of patients will benefit the most with RFA treatment.

condition [7]. While the pathogenesis of GAVE is unknown, GAVE is seen in up to $30 \%$ of patients with cirrhosis. It can account for up to $4 \%$ of nonvariceal upper gastrointestinal bleeding [8]. In non-cirrhotic patients, GAVE has been associated with various autoimmune conditions (62\%), most commonly Raynaud's phenomenon (31\%) [9].

Various treatment modalities, including surgical antrectomy, different pharmacological therapies, and endoscopic thermal techniques like Nd:Yag laser and argon plasma coagulation (APC) have been tested with mixed results. Two recent studies evaluated the efficacy of radiofrequency ablation (RFA) therapy with the HALO90 Ablation Catheter System (Covidien, GI Solutions, Sunnyvale, CA) for treatment of GAVE. In a pilot study of six patients treated with the HALO90 ablation system [1], average hemoglobin increased from $8.6 \mathrm{~g} / \mathrm{dL}$ pre-ablation to $10.2 \mathrm{~g} / \mathrm{dL}$ at two months post-ablation. Five of six patients with GAVE who received RFA (86\%) were transfusion-free at a mean follow-up of two months. In a subsequent 
study of 21 patients [10], 86\% (18/21) of patients were transfusion-free at six months and did not have any endoscopic evidence of GAVE. Mean hemoglobin increased from $7.8 \mathrm{~g} / \mathrm{dL}$ to $10.2 \mathrm{~g} / \mathrm{dL}$ in those 18 patients $(P<0.001)$. Here, we describe our experience with the use of RFA therapy in treating patients with refractory GAVE.

\section{Methods \\ $\nabla$}

\section{Study design}

This study was approved by the Institutional Review Board at University of Texas Health Science Center at Houston. Informed consent was obtained from each patient prior to the procedure. Patients included in the study were those who had endoscopic findings consistent with GAVE. All of the patients had previously undergone endoscopic biopsy confirming GAVE and received subsequent therapy with APC but continued to require blood transfusions. Thorough history was performed and sex, age, and presence of GAVE-associated chronic medical conditions were noted. Pre-and post-treatment hemoglobin levels and the number of RFA sessions were recorded. RFA was repeated if a followup visit revealed persistent gastrointestinal bleeding and symptomatic anemia. Technical success was defined as the ability to perform complete endoscopic ablation of GAVE lesions. Clinical success was defined as the patient being transfusion-free at a follow-up examination.

\section{Procedure}

All endoscopic procedures were performed in the outpatient endoscopy suite. Endoscopy was performed with the HALO90 Ablation Catheter System (Covidien, GI Solutions, Sunnyvale, California). After initial inspection of the GAVE lesions, the endoscope was removed and fitted with the ablation device at the 12 o'clock position. The endoscope was then advanced to the pylorus and the lesions were washed with Mucomyst. The endoscope was deflected upward and adjusted such that the electrode was in full contact with the affected tissue. GAVE lesions were treated until completely ablated at an automated energy density dose of $12 \mathrm{~J} / \mathrm{cm}^{2}$ and power density of $40 \mathrm{~W} / \mathrm{cm}^{2}$. Two "pulses" (application of HALO electrode to gastric mucosa with delivery of radiofrequency energy) were routinely used to ablate the GAVE tissue ( $\bullet$ Video $\mathbf{1})$. All procedures were performed in the outpatient setting. Patients were allowed to have a liquid diet immediately after the procedure once fully awake and diet was advanced as tolerated on the same day. Patients were started on proton pump inhibitor therapy for $6-8$ weeks.

\section{Statistical analysis}

For continuous variables, the results were expressed by using mean \pm standard deviation or median (interquartile range; IQR) for skewed data. Frequencies (\%) were used for categorical variables. Reported $P$-values were two-sided and all comparisons attained statistical significance at $P<0.05$. All analysis was per-

\section{Video 1}

RFA for GAVE. formed using Stata Statistical Software: Release 12, StataCorp LP, College Station, Texas.

\section{Results}

Seven patients underwent RFA for treatment of refractory GAVE. Most patients, 6/7 (87\%), were women. The mean age was 69 (range: 56-82). Two patients had a history of chronic hepatitis C with liver cirrhosis. One of these two patients had a history of hypothyroidism, while the other patient had a history of systemic lupus erythematosus. Three patients had a history of scleroderma and one patient had a history of hypothyroidism. In one patient, a 79-year-old man, there was no known history of an identifiable disease associated with GAVE ( $\bullet$ Table 1 ). All of the patients had previously undergone at least three endoscopic therapies with APC.

Three different endoscopists performed a total of twelve RFA procedures for these seven patients. 0 Fig. 1 a and $\boldsymbol{O}$ Fig. $\mathbf{1 b}$ show endoscopic images of GAVE before and after RFA, respectively. The median number of RFA treatments per patient was 2 (range, $1-3$ ). Two patients required only a single session of RFA. Four patients were treated with two sessions of RFA treatment and one patient required three sessions of RFA. RFA was successfully completed in all patients without any complication and the technical success rate was determined to be $100 \%$. Mean pre- and postprocedural hemoglobin values were $9.3 \mathrm{~g} / \mathrm{dL}$ (SD, 0.78) and $10.16 \mathrm{~g} / \mathrm{dL}$ (SD, 1.72) $(P>0.05)$, respectively. Overall, these seven patients were followed for a median of six months (range, 1.5 -8 months). Five patients ( $71 \%$ ) were transfusion-free and two patients $(29 \%)$ continued to require post-procedural transfusions at median follow-up of 6 months. Thus, the clinical success rate was determined to be $71 \%$. There were no procedure-related complications ( $\bullet$ Table $\mathbf{1}$ ).

One patient, a 56-year-old woman with history of hypothyroidism, hepatitis $\mathrm{C}$, and cirrhosis, continued to have persistent occult

\begin{tabular}{|lll}
\hline $\begin{array}{l}\text { Table } 1 \text { Patient demographics and results. } \\
\text { Patient demographics }\end{array}$ & \\
\hline Sex & Number & Percent \\
\hline Men & 1 & 14 \\
Women & 6 & 86 \\
\hline Age (years) & Mean & SD; Range \\
\hline RFA sessions (number) & 69.14 & $10.82 ; 56-82$ \\
\hline & Median & Range \\
\hline RFA "Pulse" per session (number) & 2 & $1-3$ \\
\hline & Median & Range \\
\hline Results & 42 & $16-84$ \\
\hline Success & & \\
\hline Technical & Number & Percent \\
\hline Clinical & $7 / 7$ & 100 \\
\hline Hemoglobin (g/dL) & $5 / 7$ & 71 \\
\hline Pre-RFA & Mean & SD \\
\hline Post-RFA & 9.3 & 0.78 \\
\hline Follow up (months) & 10.16 & 1.72 \\
\hline GAVE-associated conditions & Median & Range \\
Cirrhosis & 5 & $4-8$ \\
\hline Hepatitis C & Number & Percent \\
Scleroderma & 2 & 29 \\
Hypothyroidism & 2 & 29 \\
Systemic lupus erythematosus & 2 & 29 \\
\hline
\end{tabular}




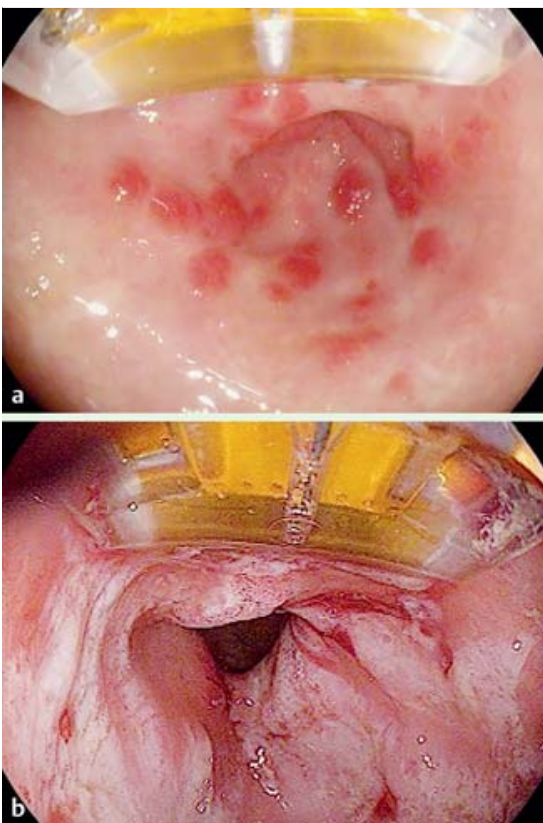

Fig. 1 a Pre-RFA appearance of GAVE. b Post-RFA appearance of GAVE. gastrointestinal bleeding with transfusion-dependent iron deficiency anemia. This patient subsequently underwent endoscopy with APC. One additional patient, an 80-year-old woman with history of hypothyroidism, GAVE, valvular heart disease, and multiple AVMs in jejunum, continued to have transfusion-dependent anemia despite three RFA treatments for GAVE. She had undergone prior double-balloon enteroscopies with APC treatment for AVMs. Her anemia was thought to be caused by a combination of bleeding from both GAVE and jejunal AVMs. She was treated with blood transfusions as needed.

\section{Discussion}

GAVE is a rare, but potentially serious, cause of gastrointestinal bleeding. Many treatment options have been proposed for GAVE. Surgical options include antrectomy, but this carries high morbidity and mortality rates $[5,6]$. Medications like octreotide, estrogen/progesterone therapy, and corticosteroids have been used, but none of these therapies have consistently reduced the number of blood transfusions needed or resolved the underlying vascular lesions $[8,10]$. Previous studies with endoscopic thermal techniques like Nd:Yag laser therapy and APC $[6,10]$ showed mixed results. Due to ease of use, decreased cost, and improved safety, APC is more commonly used than laser therapy. However, a subset of patients continue to have persistent gastrointestinal bleeding despite multiple APC treatments and RFA might be an alternative treatment for this subset of refractory GAVE patients. The HALO90 RFA system includes an endoscope-mounted ablation catheter and energy generator. The upper surface of the ablation device contains an electrode array spanning $10 \mathrm{~mm}$ long and $13 \mathrm{~mm}$ wide. When the energy generator is activated by the operator, radiofrequency energy is automatically delivered to the electrode array. Because of high-power density and a pre-set energy density, uniform tissue penetration can be achieved and operator-dependent effects eliminated. Consequently, the submu- cosal layer is less likely to be injured [11]. Two previous studies showed the safety and efficacy of RFA in treating GAVE $[1,10]$. In line with these studies, our study suggests that RFA is an effective alternative to APC for treatment of refractory GAVE. At median follow-up of 6 months (range, 1.5-8 months), five of seven patients (71\%) were transfusion-free after RFA treatment. The RFA catheter ablates a uniform area per given application and allows a larger area of ablation than APC treatment alone, which is more dependent upon the physician's skill and comfort with the technique. In addition, APC can result in intermittent skip areas between the treated areas.

Study patients had GAVE-associated chronic conditions, as documented in previous studies [1,3-6,10]. Although a direct correlation between chronic disease severity and severity of GAVE has not been established, symptomatic patients may benefit the most from RFA treatment combined with appropriate disease management.

Our study has several limitations. First, only a small subset of patients was evaluated and the results may not correlate directly with a larger patient population. Additionally, there was a relatively short follow-up (median, 6 months; range, 1.5 - 8 months); thus, the long-term efficacy of RFA could not be definitively established. Despite these limitations, findings suggest that RFA is a valuable tool in treating GAVE refractory to other endoscopic methods and merits additional evaluation. Future studies examining long-term effects of RFA and the association of GAVE with chronic medical conditions are needed to identify the subsets of patients that will benefit from RFA treatment.

\section{Competing interest: None}

\section{References}

1 Gross SA, Al-Haddad M, Gill KR et al. Endoscopic mucosal ablation for the treatment of gastric antral vascular ectasia with the HALO90 system: a pilot study. Gastrointest Endosc 2008; 67: 324-327

2 Rider JA, Klotz AP, Kirsner JB. Gastritis with veno-capillary ectasia as a source of massive gastric hemorrhage. Gastroenterology 1953; 24: $118-123$

3 Kar P, Mitra S, Resnick JM et al. Gastric antral vascular ectasia: case report and review of the literature. Clin Med Res 2013; 11: 80-85

4 Fuccio L, Mussetto A, Laterza L et al. Diagnosis and management of gastric antral vascular ectasia. World J Gastrointest Endosc 2013; 5: 6-13

5 Selinger CP, Ang YS. Gastric antral vascular ectasia (GAVE): an update on clinical presentation, pathophysiology and treatment. Digestion 2008; 77: $131-137$

6 Sebastian S, O'Morain CA, Buckley MJ. Review article: current therapeutic options for gastric antral vascular ectasia. Aliment Pharmacol Ther 2003; 18: $157-165$

7 Novitsky YW, Kercher KW, Czerniach DR et al. Watermelon stomach: pathophysiology, diagnosis, and management. J Gastrointest Surg 2003; 7: 652-661

8 Dulai GS, Jensen DM, Kovacs TO et al. Endoscopic treatment outcomes in watermelon stomach patients with and without portal hypertension. Endoscopy 2004; 36: 68 - 72

9 Gostout CJ, Viggiano TR, Ahlquist DA et al. The clinical and endoscopic spectrum of the watermelon stomach. J Clin Gastroenterol 1992; 15: $256-263$

10 McGorisk T, Krishnan K, Keefer $L$ et al. Radiofrequency ablation for refractory gastric antral vascular ectasia (with video). Gastrointest Endosc 2013; 78: 584-588

11 Pouw RE, Sharma VK, Bergman JJ et al. Radiofrequency ablation for total Barrett's eradication: a description of the endoscopic technique, its clinical results and future prospects. Endoscopy 2008; 40: 1033-1040 